\title{
Local Dynamics of Bulk Polybutadienes of Various Microstructures: Comparison of Theory with NMR Measurements
}

\section{Baysal, B. Erman, and I. Bahar*}

Polymer Research Center, School of Engineering, Bogazici University, and TUBITAK Advanced Polymeric Materials Research Center, Bebek 80815, I stanbul, Turkey

\section{F. Lauprêtre and L. Monnerie}

Laboratoi re de Physi cochimie Structurale et Macromoléculaire associé au C.N.R.S., Ecole Supérieure de Physique et Chimie Industrielles de la Ville de Paris, 10 Rue Vauquelin, F-75231 Cedex 05, Paris, France

Received March 28, $1996^{\otimes}$

\begin{abstract}
Theoretical and experimental results are presented for the local dynamics of bulk polybutadienes (PB) of various microstructures, i.e., various fractions of cis and trans units. The cooperative kinematics (CK) method is used to analyze the specific mechanism of motion involved in the rotational isomerization of the bonds belonging to the cis and trans units. Correlation times measured by NMR experiments at room temperature are reported for various mi crostructures and interpreted using the CK model. The effective monomeric friction coefficient and its dependence on the microstructure of PB are seen to dominate the rate of local motions. On the other hand, the specific mechanism of relaxation shows little dependence on the frictional resistance or on the composition of the polymer, but is uniquely defined depending on the given conformational state, cis or trans, of the double bond of the repeat unit. The amplitude of the spatial reorientations of the methylene $\mathrm{C}-\mathrm{H}$ bond vectors and the frequencies of isomeric jumps are the two contributions affecting the observed correlation times in NMR experiments. The frequencies of isomeric jumps are comparable in cis and trans units, in general. On the other hand, the $\mathrm{C}-\mathrm{H}$ bonds belonging to cis units are shown to undergo significantly larger amplitude reorientations during the cooperative motions accompanying bond isomerization, compared to $\mathrm{C}-\mathrm{H}$ bonds in trans units. This latter effect explains the differences in the NMR correlation times of cis and trans units in the chains of various microstructures.
\end{abstract}

\section{Introduction}

Local dynamics of bulk polymers at temperatures well above the glass transition temperature involve the cooperative motions of a finite number of bonds along the chain. These motions consist of rotational jumps of bonds from one isomeric state to another, librations of the bond dihedral angles, and fluctuations of bond angles and bond lengths. Among these, the rotational isomeric jumps constitute the major mechanism of local dynamics. ${ }^{1}$ Torsional librations, on the other hand, have cooperative components that assist the isomeric jumps by accommodating and localizing the conformational changes induced by rotational transitions. ${ }^{2}$ Thus, motions of chain segments of 8-12 bonds become possible in a constrained environment without invoking the motion of the points of the chain distant along the contour.

Characteristics that describe local dynamics are correlation times associated with orientational motions, activation energies, the amplitude of the motion, and the size of the chain segment which is involved in a cooperative conformational transition. On the theoretical side, these parameters may be clarified for specific polymers in the bulk state by molecular dynamics (MD) simulations, at the expense of extensive computational time and memory. Experimentally, ${ }^{13} \mathrm{C}$ NMR spectroscopy is presently the most suitable technique due to its high resolution and selectivity.

The understanding of the mechanism of Iocal conformational motions is especially important because of the involvement of such motions in the glass transition. In

\footnotetext{
${ }^{\otimes}$ Abstract published in AdvanceACS Abstracts, March 1, 1997.
}

an attempt to gain an understanding of the mechanism of local motions, a theoretical model and method, referred to as cooperative kinematics (CK), has been recently developed in a series of papers. ${ }^{3-6}$ This is a variational method in which the optimal geometric response of chain segments of $n$ bonds, or $n+1$ atoms, to a given perturbation, such as chain extension or bond rotational isomerization, is determined by the simultaneous solution of $3(n+1)$ equations, which account for both the internal and external degrees of freedom. Comparison of the predictions of the CK theory with simulations ${ }^{7-11}$ lends support to the use of the CK method as a highly efficient tool for elucidating the geometry and energetics of the collective motions in polymers.

Recently, the conformational kinematics of cis- and trans-polybutadiene (PB) chains were investigated by the CK method. ${ }^{6}$ Attention was focused on the effects of the departure from tetrahedral backbone geometry and differences in torsional potentials of backbone bonds. Strongly correlated torsions between near neighboring bonds were shown to take place in PB. NMR measurements of spin-lattice relaxation times in solution indicated that the motions cover local segments up to three monomeric units. ${ }^{12}$ Previous CK analysis of all cis- and all trans-PB chains revealed that the mechanism of motion is unique for each type of rotating bond for cis and trans structures. ${ }^{6}$ For example, in trans-PB, second neighboring bonds separated by a double bond were shown to undergo counterrotations with higher probability, whereas in cis-PB, the same pair of bonds were subject to coupled corotations. Under the same frictional environment, the atomic displace- 
ments accompanying bond rotational jumps in cis-PB were shown to be larger than those of trans-PB by a factor of $\sim 1.5$. The predictions of the theory were in good agreement with MD results. ${ }^{9,10}$ More interestingly, good agreement between the results obtained for cis$\mathrm{PB}$ and those from the bulk state MD simulations of cis-polyisoprene $\mathrm{e}^{11}$ were found, indicating the major role played by backbone geometry in determining the mechanism of local motions. The frictional effect, on the other hand, and the torsional energy barriers to be surmounted during isomeric transitions are shown to affect the amplitude of the motions without significantly modifying the mechanism. ${ }^{5,6}$

In the present study, theoretical and experimental results will be presented on the local dynamics of PB chains of various cis and trans contents. These are referred to as PBs of various microstructures or compositions. The fraction of cis monomers is denoted as $x_{c}$. The CK method is used to analyze the specific mechanism of motion involved in the rotational isomerization of the bonds belonging to different monomeric units. Attention will be focused on the effect of composition. It will be demonstrated that the mechanism of relaxation is a property intrinsic to the conformational state, cis or trans, of the repeat unit including the rotating bond, irrespective of the microstructure of the adjoining monomeric units. On the experimental side, correlation times measured by NMR experiments will be reported for various compositions at room temperature, and interpreted in terms of the CK theory.

\section{Methods: Calculations and Experiments}

Application of the CK Approach to Polybutadienes of Different Microstructures. Each PB repeat unit comprises three rotatable bonds, $\alpha_{1}, \beta$, and $\alpha_{2}$, as shown below for a chain segment between two double bonds:

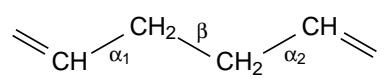

Throughout the present study, bond lengths and bond angles will be held fixed at their equilibrium values. The dihedral angles of the rotatable bonds represent the variables defining a given conformation. Thus, changes in the conformation result from changes in the torsional angles, only. The double bonds are constrained to assume either the cis or the trans state, depending on the selected geometric isomer, by a severe potential favoring either of these geometric isomers.

In the CK approach, the conformational motions are obtained by a succession of small incremental changes occurring collectively in the dihedral angles. The total energy change $\Delta \mathrm{E}$ involved in a small conformational change is taken as the sum of two terms: (i) the work done against friction $\delta \mathrm{W}_{\xi}$ by the chain atoms being displaced and (ii) the change $\Delta \mathrm{V}$ in the total conformational energy of the chain. As in previous studies, ${ }^{3-6}$ an internal bond, starting originally at a rotational isomeric minimum, is given a small torsion while the chain is subjected to the condition that the total energy change $\Delta \mathrm{E}$ is a minimum. The accompanying changes in the other torsional angles $\Delta \varphi_{\text {i }}$, in the absolute position (or orientation) of chain atoms (or bonds) are determined. This condition is expressed by a set of $n+3$ equations including the differentiations with respect to dihedral angles

$$
\frac{\partial \Delta \mathrm{E}}{\partial \Delta \varphi_{\mathrm{i}}} \equiv \frac{\partial\left[\delta \mathrm{W}_{\xi} / 2+\Delta \mathrm{V}\right]}{\partial \Delta \varphi_{\mathrm{i}}}=0
$$

for $2 \leq \mathrm{i} \leq \mathrm{n}-1$, and six additional equations, not presented here for brevity, containing the derivatives with respect to the variables $\left[\mathrm{X}_{0}, \mathrm{Y}_{0}, \mathrm{Z}_{0}, \varphi_{1}, \Phi\right.$, and $\left.\Psi\right]$ which define the spatial position and orientation of the overall chain. In eq $1, \delta \mathrm{W}_{\xi}$ is the work done against friction by all atoms moving during a small configurational change, $\varphi_{\mathrm{i}}$ is the dihedral angle of the ith bond connecting backbone atoms $i-1$ and $i$, and atoms are indexed from 0 to $\mathrm{n}$. $\delta \mathrm{W}_{\xi}$ is related to the monomeric friction coefficient $\xi$ of the medium by the expression

$$
\delta \mathrm{W}_{\xi}=(\xi / \delta \mathrm{t}) \sum_{\mathrm{i}} \delta \mathbf{R}_{\mathrm{i}} \cdot \delta \mathbf{R}_{\mathrm{i}}
$$

where $\delta \mathbf{R}_{\mathrm{i}}$ is the change in the position vector $\mathbf{R}_{\mathrm{i}}$ of the ith atom and $\delta$ is the time interval during which this change takes place. The summation is carried over all $\mathrm{n}+1$ atoms of the chain. The total conformational energy $V$ of the chain is obtained from the summation of the rotational potential $\mathrm{V}_{\varphi}\left(\cos \varphi_{\mathrm{i}}\right)$ of individual bonds, assuming bond torsional potentials to be independent. $\mathrm{V}_{\varphi}\left(\cos \varphi_{\mathrm{i}}\right)$ is taken as a fifth order polynomial in powers of $\cos \varphi_{i}$, as

$$
\mathrm{V}=\sum_{\mathrm{i}=2}^{\mathrm{n}-1} \mathrm{v}_{\varphi}\left(\cos \varphi_{\mathrm{i}}\right)=\mathrm{k}_{\varphi} \sum_{\mathrm{i}=2}^{\mathrm{n}-1} \sum_{\mathrm{m}=0}^{5} \mathrm{a}_{\mathrm{im}} \cos ^{\mathrm{m}} \varphi_{\mathrm{i}}
$$

The parameter $k_{\varphi}$ and the coefficients $a_{i 0}, \ldots, a_{i 5}$ for each type $i$ of bond in cis- and trans-PB are listed in our previous work. ${ }^{6}$

The combined effect of the height of energy barriers $\mathrm{k}_{\varphi}$, the friction coefficient $\xi$, the length I of the rotating bond, and the time $\delta$ t associated with the incremental changes in dihedral angles are conveniently described in terms of a coefficient $k_{0} a s^{5,6}$

$$
\mathrm{k}_{0}=\frac{2 \mathrm{k}_{\varphi}}{\mathrm{I}^{2} \xi / \delta \mathrm{t}}
$$

This ratio provides a measure of the relative strength of the two effects, intermolecular and intramolecular, in selecting a particular mechanism of motion. In fact, for fixed I and $\delta \mathrm{t}$, the numerator and the denominator of eq 4 reflect the strength of the internal torsional barriers and the frictional resistance, respectively.

The value of the effective friction coefficient $\xi\left(x_{C}\right)$ depends on the microstructure of the PB chains. A convenient form that may be deduced from the experiments of Chen and Ferry ${ }^{13}$ is

$$
\log \xi\left(x_{c}\right)=A+B\left(1-x_{c}\right)^{2}
$$

This form is adopted in the present work, with the coefficients $A$ and $B$ evaluated by least-squares fit to experimental data. In addition, in a given copolymer, all monomers are assumed to experience the same effective friction coefficient. $\xi\left(x_{c}\right)$ is obtained from the experimental diffusivities $D\left(x_{c}\right)$, using the relation $\xi\left(x_{c}\right)$ $=\mathrm{kT} / \mathrm{D}\left(\mathrm{x}_{\mathrm{c}}\right)$. The parameters $\mathrm{A}$ and $\mathrm{B}$ for $\mathrm{PB}$ in eq 5 are taken as -7.0 and 1.0, respectively, $\xi\left(x_{c}\right)$ being expressed in dyn/(cm.s), using the least-squares fit of the data provided by Chen and Ferry, and more recent rheological measurements carried out by Bokobza et al. ${ }^{14}$ 
Table 1. Code Names, Microstructures, and Glass Transition Temperatures of the Samples Investigated

\begin{tabular}{ccccc}
\hline code name & \% vinyl-1,2 & \% cis- 1,4 & $\%$ trans- 1,4 & $\mathrm{~T}_{\mathrm{g}}\left({ }^{\circ} \mathrm{C}\right)$ \\
\hline PB17 & 4 & 17 & 79 & -85 \\
PB32 & 4 & 32 & 64 & -101 \\
PB75 & 4 & 75 & 21 & -106 \\
PB93 & 4 & 93 & 3 & -106
\end{tabular}

Equations 1-5 give the outline of the CK method as applicable to PB chains with different microstructures. For more details including the explicit forms of the $n+$ 4 linearized equations used in calculations, and their solution in matrix notation, the reader is referred to our previous work.5,6

The following approach is adopted in calculations: For each given cis fraction $x_{c}, 500$ PB chains of different cis/ trans sequence and original configuration are generated subject to the conditions described by $\mathrm{k}_{0}$. Interdependence of the rotational behavior of the bonds $\alpha_{1}$ and $\alpha_{2}$ separated by a double bond is accounted for in the generation of the original structures via the statistical weight matrices of Abe and Flory. ${ }^{15}$ An internal bond is rotated at small steps until a transition from one isomeric state to another is completed. At each step, the minimization given by eq 1 is performed numerically, and the values $\Delta \mathrm{E}, \delta \mathrm{W}, \Delta \mathrm{V}, \varphi_{\mathrm{i}}$, and $\mathbf{R}_{\mathrm{i}}$ are determined. Long-range interactions are also examined at the initial and intermediate incremental rotation steps, and those configurations viol ating an interatomic cutoff distance of $3.8 \AA$ are discarded.

${ }^{13} \mathrm{C}$ NMR Experiments on Bulk Polybutadienes with Different Microstructures. The PB samples were kindly supplied by Michelin. Their microstructure was determined by ${ }^{13} \mathrm{C}$ NMR in solution, using either the olefinic resonances according to ref 16 or the aliphatic lines according to refs 17 and 18, which did not result in significant differences. NMR values derived for samples with various contents of cis-1,4, trans1,4 and vinyl-1,2 units are shown in Table 1, together with the code names and glass transition temperatures, $\mathrm{T}_{\mathrm{g}}$, of the polymers.

${ }^{13} \mathrm{C}$ NMR spectra were recorded at 25 and $50 \mathrm{MHz}$ on a Bruker AC-100 and a Bruker AC-200 spectrometer, respectively, using proton noise decoupling. ${ }^{13} \mathrm{C}$ spinlattice relaxation times, $T_{1}$, were measured using the standard $\left(180^{\circ}, \mathrm{t}, 90^{\circ}\right)$ pulse sequence, with a $10 \mu \mathrm{s} 90^{\circ}$ pulse duration and repetition times between pulse sequences greater than 5 times the longest $T_{1}$ of the considered nuclei. The accuracy for each $T_{1}$ measure ment is of the order of $\pm 0.05 \mathrm{~s}$.

The correlation time $\tau_{1}$ determined by ${ }^{13} \mathrm{C} N M R$ experiments is deduced from the time decay of the second orientational autocorrelation function (OACF) of the methylene $\mathrm{C}-\mathrm{H}$ bond vectors belonging to cis or trans units. The second $\mathrm{OACF}, \mathrm{M}_{2}(\mathrm{t})$, is given by the expression

$$
M_{2}(t)=\frac{1}{2}\left[3\left\langle\cos ^{2} \theta(t)\right\rangle-1\right]
$$

where $\theta(\mathrm{t})$ is the angle between two orientations of the examined $\mathrm{C}-\mathrm{H}$ bond vector at times 0 and $\mathrm{t}$. The angular brackets denote an ensemble average. The classical method to extract a correlation time from the time decay of the OACF is to take the integral of the OACF as

$$
\tau_{c}=\int \frac{M_{2}(t)-M_{2}(\infty)}{M_{2}(0)-M_{2}(\infty)} d t
$$

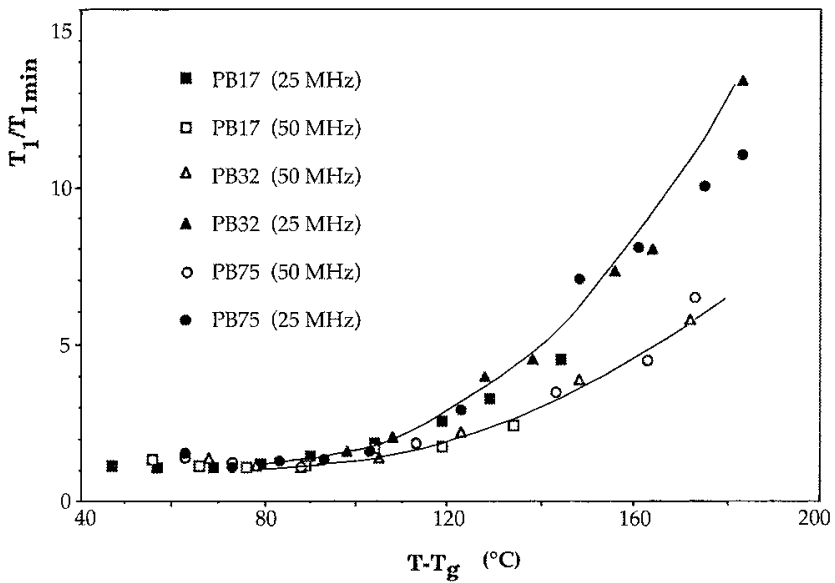

Figure 1. Dependence of $T_{1} / T_{1 m i n}$ on the temperature difference $\mathrm{T}-\mathrm{T}_{g}$, determined at 25 and $50 \mathrm{MHz}$, for methylene carbons of trans-1,4 units. Results for chains of various microstructures are displayed, as indicated on the figure. The best fitting curves obtained using eq 8 are also displayed.

The correlation times $\tau_{1}$ presently derived from NMR data are obtained using the four-parameter empirical expression

$$
\begin{array}{r}
M_{2}(t)=(1-a) \exp \left(-t / \tau_{2}\right) \exp \left(-t / \tau_{1}\right) I_{0}\left(t / \tau_{1}\right)+ \\
a \exp \left(-t / \tau_{3}\right)
\end{array}
$$

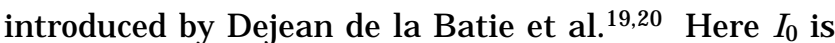
the modified Bessel function of order zero, $\tau_{1}$ is the characteristic time responsible for the diffusion of bond orientation al ong the chain, and $\tau_{2}$ is the damping term corresponding to the orientation loss processes. $\tau_{3}$ is the characteristic time for librations. a is related to the half-angle of the libration cone. For fast librations and segmental motions, the second term in eq 8 may be neglected. Under this condition, the $\mathrm{T}_{1}$ value at the minimum, $T_{1 \mathrm{~min}}$, is directly proportional to $1 /(1-a)$ and the $T_{1} / T_{1 \min }$ ratio describes the segmental motions only. Results obtained for cis-1,4-PB ${ }^{19}$ show that the olefinic and methylene carbons share the same local segmental dynamics in terms of $\tau_{1}$ and $\tau_{2}$ correlation times, and different libration amplitudes, which violates the NT rule, where $\mathrm{N}$ is the number of protons directly bound to the carbon of interest. ${ }^{21}$ In the case of the PB samples considered in this work, the ${ }^{13} \mathrm{C}$ NMR lines of the ol efinic carbons (131 ppm) of the cis and trans units were not resolved. On the contrary, the methylene carbons of the cis and trans units exhibited two distinct resonances at 29 and 34 ppm, respectively. Therefore we use the data obtained from the methylene resonances to investigate the segmental dynamics of each unit separately. For illustrative purposes, the dependence of $\mathrm{T}_{1} / \mathrm{T}_{1 \mathrm{~min}}$ on the temperature difference $T-T_{g}$, determined at 25 and $50 \mathrm{MHz}$ is shown in Figure 1, for methylene carbons of trans-1,4 units. The best fitting lines obtained using eq 8 are also displayed. The corresponding $\tau_{1}$ values are displayed in Figure 2 as a function of temperature. The curves are drawn through the points to guide the eye. In obtaining this figure, $\tau_{2} / \tau_{1}$ is taken as 30 over the whole temperature range. Here, our objective being to study the dependence of the local dynamics on microstructure, we will concentrate on the correlation times measured at a fixed temperature, $298 \mathrm{~K}$, as a function of $x_{c}$. The complete data, as well as the discussion of the temperature dependence will be published in a forthcoming paper.22

Formulation of Correlation Times in the CK Method. There are essentially two contributions af- 


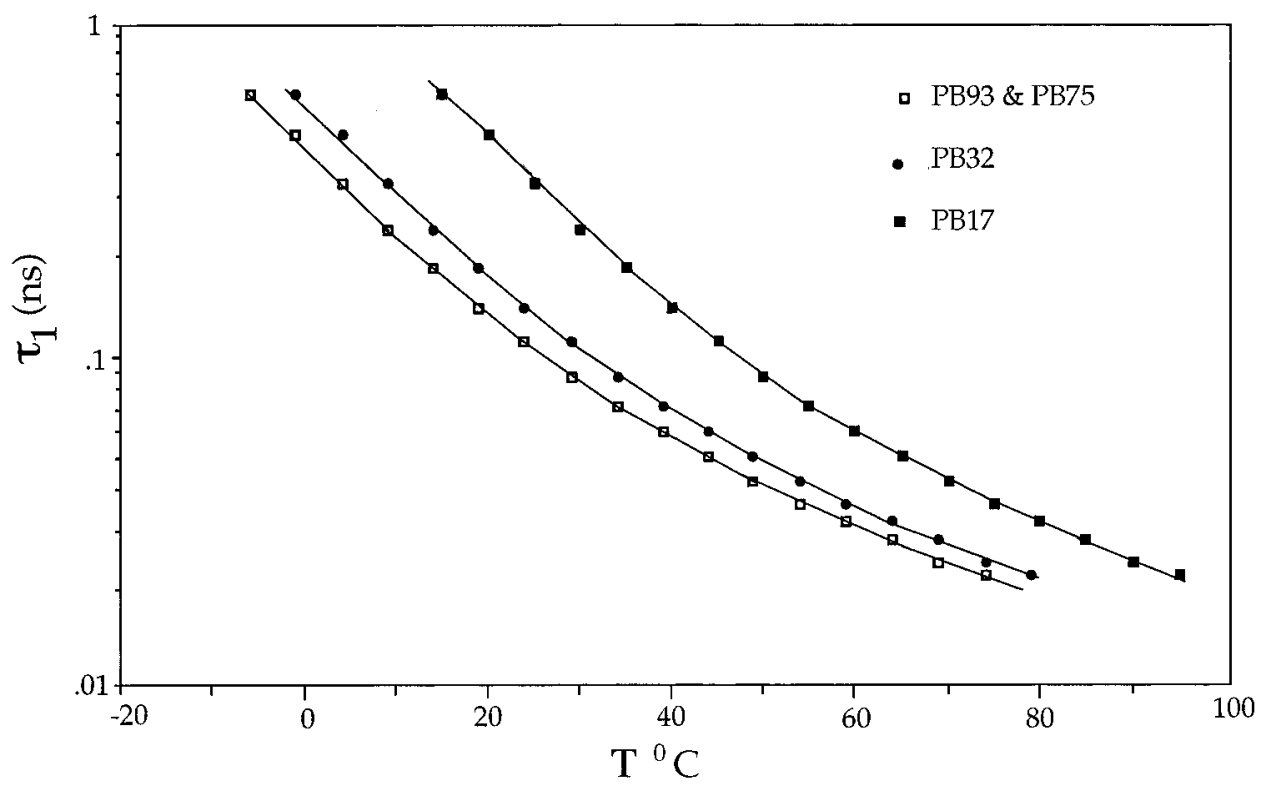

Figure 2. $\tau_{1}$ values obtained from the results of Figure 1 presented as a function of temperature. The curves are drawn through the points to guide the eye. In obtaining this figure, $\tau_{2} / \tau_{1}$ is taken as 30 over the whole temperature range.

fecting the observed $\tau_{1}$ in NMR experiments. The first is associated with the amplitude $\Delta \theta$ of the angular displacements of the $\mathrm{C}-\mathrm{H}$ bond vectors induced by isomeric jumps, larger amplitude motions resulting in a faster loss of the original orientation or in a lower $\tau_{1}$. The second factor contributing to the decay rate of the OACFs is the frequency of isomeric jumps, with the obvious effect that more frequent transitions result in shorter $\tau_{1}$. These two factors will be analyzed in the light of the theoretical results obtained by the CK method.

The amplitude of reorientations is determined by the particular mechanism of localization of the motion triggered by the isomeric jumps. In fact, a $120^{\circ}$ torsional rotation of a backbone bond does not result in a reorientation of $109.5^{\circ}$ in the tetrahedrally bonded $\mathrm{C}-\mathrm{H}$ bonds, but is distributed on both sides of the rotating bond, its effect being closely dependent on the chemical structure of the rotating bond and of the segment set in motion. In general, a spectrum of $\Delta \theta$ values may obtain, depending strongly on the local microstructure, as will be illustrated below. The jump frequency, on the other hand, may conveniently be associated with (i) the activation energy for conformational motions following the classical rate theory, and (ii) a front term including the effect of friction and path swept by the moving atoms during local motions. Combining these effects, one may write for $\tau_{\mathrm{c}}$ a proportionality of the form

$$
\tau_{\mathrm{c}} \sim \tau_{0}(\Delta \theta)\left[\xi\left(\mathrm{x}_{\mathrm{c}}\right) \sum_{\mathrm{i}} \delta \mathbf{R}_{\mathrm{i}} \cdot \delta \mathbf{R}_{\mathrm{i}}\right] \exp [\Delta \mathrm{V} / \mathrm{RT}]
$$

where $\tau_{0}(\Delta \theta)$ reflects the contribution of the amplitude $\Delta \theta$ of the reorientation of the investigated $\mathrm{C}-\mathrm{H}$ bond, which is induced by the isomeric jumps. The last term represents the activation energy contribution, and the middle term in square brackets holds for the effect of the frictional resistance. This expression may be rewritten as

$$
\tau_{\mathrm{c}}=\mathrm{A} \tau_{0}(\Delta \theta) \mathrm{W}_{\xi} \exp [\Delta \mathrm{V} / \mathrm{RT}]
$$

where the prefactor $A$ is left as an adjustable parameter. $A$ is found from a least-squares fit between experimental data and theoretical results. Equation 2 is used in going from eq 9 to eq 10. The coefficient $\tau_{0}(\Delta \theta)$ may be viewed as the amplitude factor contributing to reorientational relaxation in the absence of friction and activation energy effects. Equation 10 is essentially equival ent to the expression based on Kramer's rate theory in the high friction limit

$$
\tau_{\mathrm{c}}=\frac{2 \pi \xi \sum_{\mathrm{i}} \delta \mathbf{R}_{\mathrm{i}} \cdot \delta \mathbf{R}_{\mathrm{i}}}{\left(\gamma \gamma^{*}\right)^{1 / 2}} \exp [\Delta \mathrm{V} / \mathrm{RT}]
$$

where $\gamma$ and $\gamma^{*}$ are the curvatures of the potential at the minimum and the maximum along the trajectory through which the transition takes place. The summation over the squared displacements in the numerator of eq 11 has been introduced ${ }^{1,23}$ to account for the frictional resistance against all moving atoms of the polymer chain. This term multiplied by the monomeric friction coefficient is identical to the summation of the $\delta \mathrm{W}_{\xi} \delta \mathrm{t}$ term of eq 2 over a complete isomerization of a bond.

The factor $\tau_{0}(\Delta \theta)$ in eq 10, being of purely geometric origin, may be estimated by analyzing the time evolution of the spatial orientations of a vector which undergoes a series of step jumps of a given angular size $\Delta \theta$. This type of motion has been considered by Torchia and Szabo analytically for the case of a nearest neighbor $\mathrm{N}$-site jump model for crystalline solids. ${ }^{24}$ In the present study, a numerical approach will be adopted for estimating $\tau_{0}(\Delta \theta)$ as a function of the jump angles $\Delta \theta$. Accordingly, a unit vector $\mathbf{m}$ representative of the methylene $\mathrm{C}-\mathrm{H}$ bond under investigation will be subjected to successive random reorientations of fixed angles in space, assuming each jump to occur at unit time. The resulting trajectory for the reorientation angle $\theta(\mathrm{t}) \equiv \cos ^{-1}[\mathbf{m}(0) \cdot \mathbf{m}(\mathrm{t})]$ is used to evaluate $\tau_{0}(\Delta \theta)$ following eqs 6 and 7 .

\section{Results of CK Calculations}

In the present section, CK results are presented for PB chains of various microstructures at $\mathrm{T}=298 \mathrm{~K}$ using eqs $1-5$. The results refer to the average behavior of 500 representative chains of 25 bonds having different 

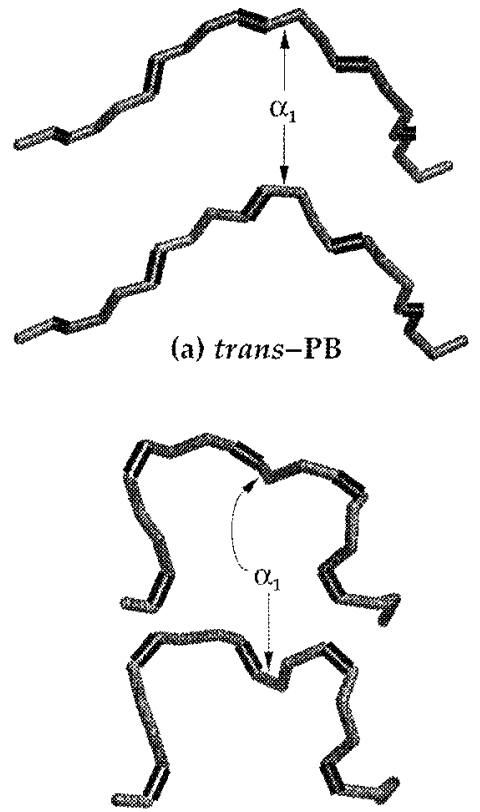

(b) cis-PB

Figure 3. Evolution of an $\alpha$-bond transition for a segment of (a) a trans-PB and (b) a cis-PB chain. The indicated bond $\alpha_{1}$ undergoes a transition from a rotational isomeric state to another in both cases. We note that the isomeric transition is localized to a few bonds only surrounding the bond $\alpha_{1}$, the orientation and position of the tails being unchanged.

original configurations and cis/trans sequences for each $x_{c}$. The results have been verified to be independent of the size of the chain, thus reflecting the mechanism of local dynamics which is confined to one to three repeat units in bulk PB. The geometry and energy parameters used in calculations, which are characteristic of the different types of bonds in cis- and trans-PB, are given in our previous work. ${ }^{2}$ The values $\delta \mathrm{t}=10 \mathrm{ps}$ and $\mathrm{I}=$ $1.53 \AA$ are used.

Relative Rates of $\alpha$ - and $\beta$-Bond I somerizations in cis- and trans-PB. It is possible to estimate the relative jump frequencies of bonds of different types in PB chains, using the CK approach, by examining the conformational energy change $\Delta \mathrm{V}$ involved in the rotational isomerizations of the particular bonds, even though time is not explicitly involved in the CK method.

For illustrative purposes, the initial and final conformations of a chain segment foll owing the isomerization of an $\alpha$ bond are shown for trans- and a cis-PB, in the respective parts $a$ and $b$ of Figure 3 . The $\alpha$ bond is in an isomeric minimum in the starting and final configurations. We note that the rotational isomerization of a central bond affects only a small segment consisting of three to four bonds on both sides of the rotating bond, while the position and orientation of the ends of the segment remain unperturbed.

In Figure 4 , the cumulative evolution of $\Delta \mathrm{V}$ in response to the rotation of the two distinct types of bonds, $\alpha$ and $\beta$, is presented for the all-cis and all-trans stereoisomers at $300 \mathrm{~K}$. The abscissa refers to the torsional rotation of $\Delta \varphi_{\mathrm{s}}$ in degrees $(\mathrm{s}=\alpha$ or $\beta$ ), starting from the initial configuration. The $\beta$ bond in trans-PB requires, on the average, an energy of about $4 \mathrm{kcal} / \mathrm{mol}$ in going from one isomeric minimum to another. This is 4 times larger than the energy to accomplish the same type of isomerization in cis-PB, even though these two bonds have the same $k_{\varphi}$ value. This difference indicates that the conformational barrier to rotation is not due to the rotating bond only, but results from the collective

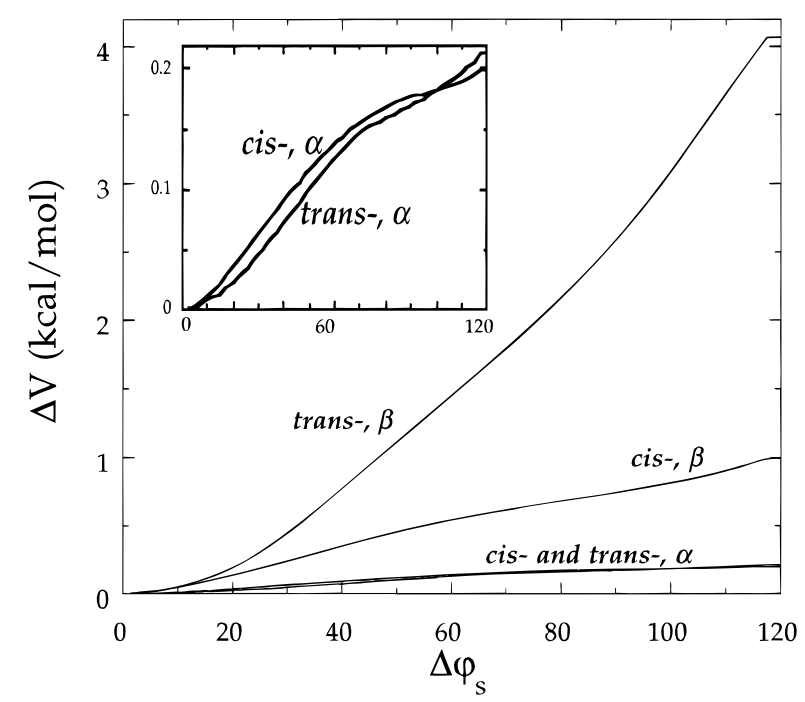

Figure 4. Cumulative conformational energy change, $\Delta \mathrm{V}$, involved in the isomerization at room temperature of (i) $\alpha$ bonds in cis-PB, (ii) $\alpha$ bonds in trans-PB, (iii) $\beta$ bonds in cis$\mathrm{PB}$, and (iv) $\beta$ bonds in trans-PB. Cases $\mathrm{i}$ and ii are magnified in the inset.

rearrangements of the neighboring bonds as well along the chain. An important observation from the figure is that the energy of isomerization of the $\alpha$ bonds of both cis- and trans-PB, which are al so enlarged in the inset, is much lower than that of the $\beta$ bonds. These results are in agreement with the MD results of Gee and Boyd ${ }^{10}$ in which $\beta$-bond transitions make up $1-5 \%$ of all transitions in trans-PB in the temperature range of $200-450 \mathrm{~K}$, whereas they amount to $10-30 \%$ in cis-PB over the same temperature range. The same results are also confirmed by the MD simulations of Kim and Mattice ${ }^{9}$ of PB in the bulk state which indicate that the difference in the torsional relaxation of the two rotatable bonds, $\alpha$ and $\beta$, is less pronounced in cis-PB. Taking into consideration the relatively low frequency of occurrence of $\beta$-bond isomerizations in PB compared to $\alpha$-bond isomerizations, we will concentrate exclusively on the isomerizations of $\alpha$ bonds in the following.

Change in Dihedral Angles Accompanying Bond I somerizations. A quantity of interest in CK calculations is the most probable change $\left\langle\Delta \varphi_{\mathrm{i}}\right\rangle$ in the dihedral angles of bonds in the neighborhood of a central bond ( $\mathrm{i}$ $=0$ ) undergoing a rotational transition between isomeric states. This provides a description of the nature of the localization process accompanying the rotational isomerization of a given bond. In Figure $5 \mathrm{a},\left\langle\Delta \varphi_{i}\right\rangle$ resulting from the isomerization of the $\alpha_{1}$ bond belonging to cis units, exclusively, is presented as a function of bond index. The identity of each type of bond is provided at the top of the figure. The curves are obtained for PB chains of different compositions $x_{c}$. The curves almost coincide with each other, revealing that the cooperative response to an $\alpha$-bond rotation in cis units is independent of the microstructure of the surrounding units al ong the chain. The same behavior is valid for the trans units in response to an $\alpha$-bond isomerization as shown in Figure 5b. The curves for PB chains of different microstructures again follow a closely similar distribution, irrespective of $x_{c}$. Figure 5 demonstrates that the rotation is highly localized and that only the stereospecific state of the monomeric unit in which the isomeric jump takes place affects the mechanism of motion; i.e., the dynamic behavior of bonds is controlled by the chemical structure of the 


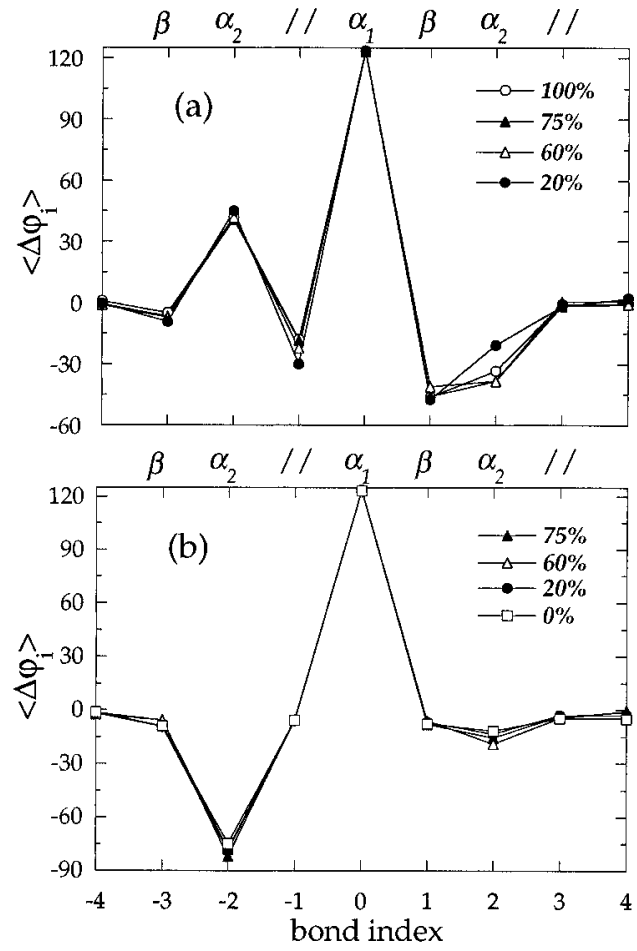

Figure 5. Average changes in the dihedral angles of bonds, $\left\langle\Delta \varphi_{i}\right\rangle$, in response to the rotational transitions of $\alpha_{1}$ bonds ( $i$ $=0)$ in PB chains with various contents of cis and trans

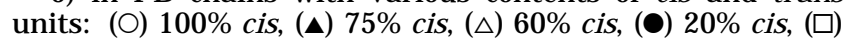
$100 \%$ trans. The identities of the rotated bond and the three bonds on either side are indicated at the top of the figure. The symbol // stands for the double bond.

repeat unit only, irrespective of the composition $x_{c}$ of the PB chains.

At a given temperature and pressure, there is a oneto-one correspondence between composition $x_{c}$ and friction coefficient $\xi$ of the medium. This relationship is indicated by eq 5 , where $\xi$ decreases monotonously with $x_{c}$. The fact that the dihedral angle distributions resulting from CK calculations are independent of $x_{c}$ for a given cis or trans unit implies that the mechanism of relaxation of the cis and/or trans units is independent of the friction coefficient and depends only on the stereospecific structure of the unit.

Atomic Displacements in Response to Bond Isomerization. Atomic displacements accompanying the isomerization of the $\alpha_{1}$ bond in a cis and a trans unit are presented in Figures 6 , parts $a$ and $b$, respectively. Calculations are performed for different microstructures, as identified in the curves. Coincidence of the curves from different microstructures in both figures confirms that the mechanism of motion depends intrinsically on the state, cis or trans, of the monomeric unit containing the rotating bond, irrespective of the state of the neighboring units in all microstructures. Overall, the displacements of atoms in a cis unit are larger than those for the trans structure. Thus, at a given monomeric friction, the amplitude of atomic motions in cis units is larger than that in trans units.

Reorientations of $\mathbf{C}-\mathbf{H}$ Bond Vectors. In previous work, we examined the reorientation of the backbone vectors $\mathrm{C}-\mathrm{C}$ for polyethylene and cis- and transPB chains. ${ }^{4-6}$ Here, inasmuch as NMR measurements are of interest, we will consider the reorientation $\Delta \theta$ of $\mathrm{C}-\mathrm{H}$ bond vectors, and particularly those of the methylene groups which are experimentally observed. The results obtained for the methylene $\mathrm{C}-\mathrm{H}$ groups at-

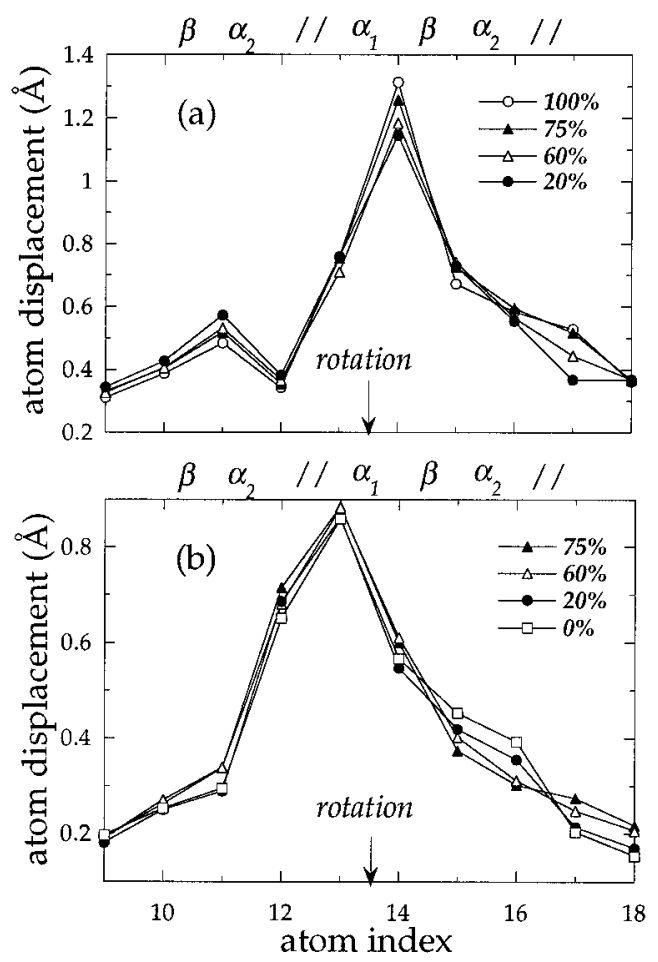

Figure 6. Atomic displacements accompanying the isomerization of the $\alpha_{1}$ bond in (a) cis units belonging to PB chains of various compositions in the range $x_{c}=20-100 \%$, as indicated. (b) trans units in chains with compositions $x_{c}=0$ $-75 \%$.
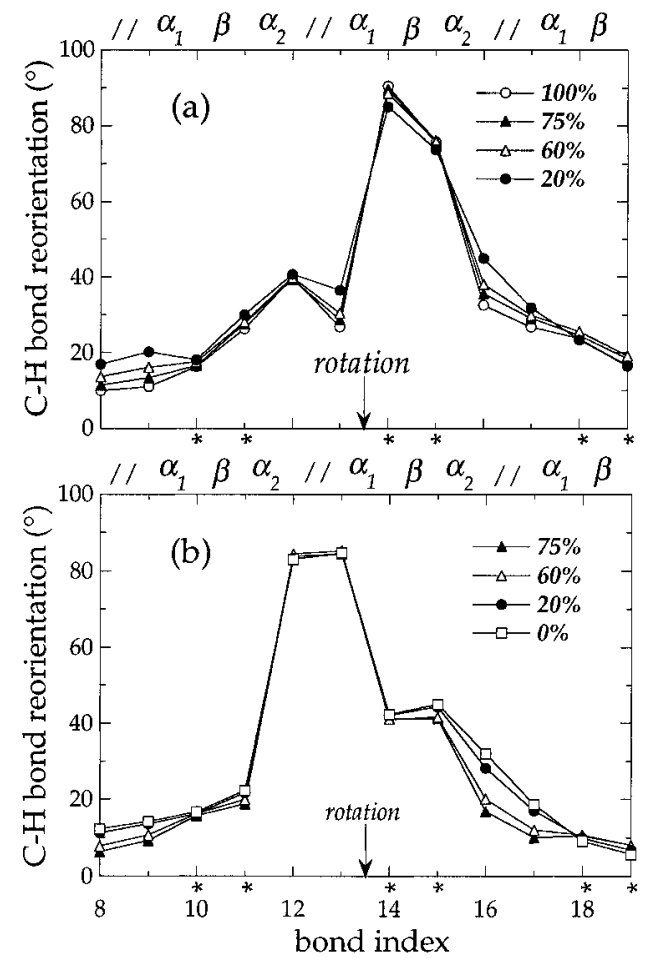

Figure 7. Reorientation of $\mathrm{C}-\mathrm{H}$ bond vectors accompanying the isomerization of the $\alpha_{1}$ bond in (a) a cis unit belonging to PB chains of various compositions in the range $x_{c}=20-100 \%$, as indicated. (b) A trans unit in PB chains of $x_{c}=0-75 \%$. Methylene $\mathrm{C}-\mathrm{H}$ bonds corresponding to atom indices 10,11 , $14,15,18$, and 19 are shown by asterisks al ong the abscissa. These are observed in NMR experiments.

tached to cis and trans $\alpha_{1}$ bonds, are presented in Figure 7 , in parallel with the treatment carried out for bond dihedral angles and atomic displacements. This figure also confirms that the angular displacements $\Delta \theta$ of the 
$\mathrm{C}-\mathrm{H}$ bonds belonging to particular monomeric units, cis or trans, depend predominantly on the state of this unit, irrespective of that of the neighbors along the chain.

In both figures, the $\alpha$ bond between atoms with indices 13 and 14, which is the middle bond of the investigated 25-bond chain, is rotated from one isomeric minimum to another, and the resulting most probable reorientations of the $\mathrm{C}-\mathrm{H}$ vectors affixed to backbone carbons 8-19 are shown. Among these, there are six pairs of methylene $\mathrm{C}-\mathrm{H}$ bonds, whose reorientations are observed by NMR. These are located at the ends of the $\beta$ bonds. The indices of the corresponding backbone atoms, shown by asterisks along the abscissa, are 10, $11,14,15,18$, and 19 . In Figure $7 a$, the largest $\Delta \theta$ in response to the $\alpha$-bond isomerization is undergone by the $\mathrm{C}-\mathrm{H}$ bonds at positions 14 and 15, flanking the $\beta$-bond. These bonds reorient by $\Delta \theta=85^{\circ}$ and $75^{\circ}$ approximately, succeeding a rotational jump. The remaining four methylene $\mathrm{C}-\mathrm{H}$ bonds undergo reorientations of $30^{\circ} \pm 10^{\circ}$. In Figure $7 \mathrm{~b}$ for trans units, on the other hand, the strongest response is that of the $\mathrm{C}-\mathrm{H}$ bonds belonging to the olefinic groups, which are not observable separately by the present NMR measurements. The six methylene $\mathrm{C}-\mathrm{H}$ bonds, whose relaxation is detected by NMR show reorientations of $43^{\circ}, 42^{\circ}, 20^{\circ}, 16^{\circ}, 9^{\circ}$, and $6^{\circ}$. As pointed out in section 2 , the amplitude $\Delta \theta$ of the reorientational jumps directly controls the term $\tau_{0}$ in eq 10. Thus, the differences in the angular displacements of the $\mathrm{C}-\mathrm{H}$ bond vectors attached to cis and trans units will be reflected upon the correlation times observed for the two units, as will be further elaborated below.

\section{Interpretation of Experimental Observations}

Calculations presented above demonstrate that the mechanism of relaxation in the neighborhood of a rotating bond depends primarily on the stereospecific state, cis or trans, of the repeat unit comprising the rotating bond, irrespective of the overall composition of the chain, and consequently of the effective friction coefficient $\xi$ which is a function of the composition. The friction coefficient plays, on the other hand, a major role in determining the absolute rate of relaxation, despite its insignificant effect on the mechanism of motion; and comparison of the CK model with experiments necessitates the proper choice of $\xi\left(x_{c}\right)$ to be inserted in eq 2 .

In order to compare the correlation times measured in NMR experiments with CK predictions based on eq 10 , we need to evaluate the changes in the conformational energy $\Delta \mathrm{V}$ and the work $\mathrm{W}_{\xi}$ performed against environment during the rotational transitions of $\alpha$ bonds and the accompanying accommodating motions. Results from CK calculations performed over a wide range of $\xi$ values, $0 \leq \xi \leq 6 \times 10^{-7} \mathrm{dyn} /(\mathrm{cm} \cdot \mathrm{s})$, are presented in Figure 8 for the respective cases of cis and the trans units comprising the rotating bond. The symbols in the figures represent the results from calculations. The curves are the polynomials best fitting the CK results, which allow for expressing the respective potentials as a function of $\xi$. The substitution of the values of $\Delta \mathrm{V}$ and $W_{\xi}$ obtained as a function of $\xi$ into eq 10 yields the relaxation time $\tau_{c}$ associated with $\alpha$-bond isomerization in cis and trans units, provided that the front factors $A$ and $\tau_{0}$ are also known. As described in section $2, \tau_{0}$ is determined from the $\Delta \theta(\mathrm{t})$ trajectories generated by simulations performed with various jump angles. The OACF obtained by adopting fixed jump angles of $40^{\circ}$
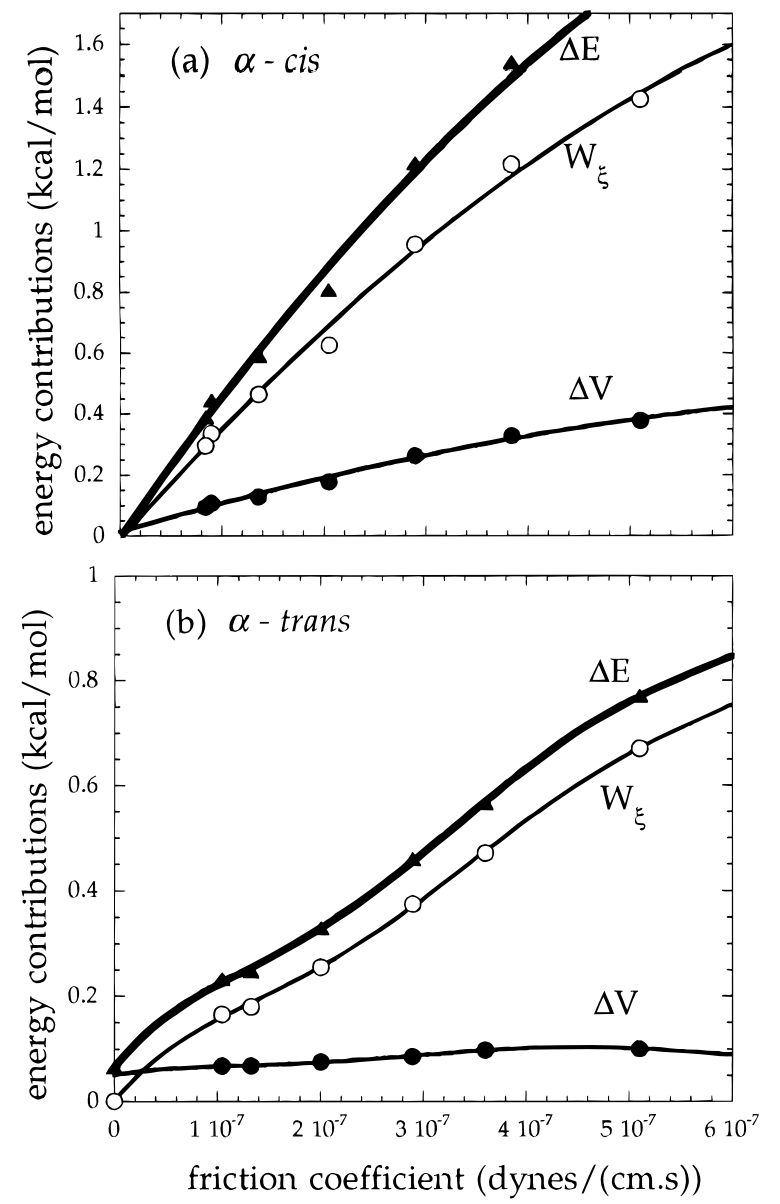

Figure 8. Energy changes $\Delta \mathrm{E}, \Delta \mathrm{V}$, and $\mathrm{W}$ involved in the isomerization of $\alpha$ bonds, obtained by the CK calculations at $298 \mathrm{~K}$ over a wide range of friction coefficients. Results are presented for $\alpha$ bonds belonging to cis and trans units in parts (a) and (b), respectively.

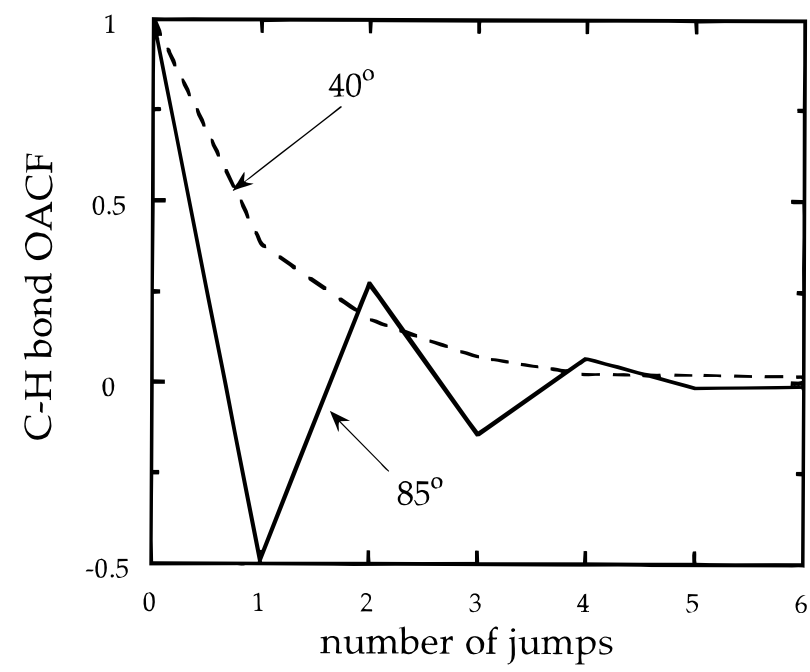

Figure 9. $O A C F$ for a unit vector undergoing angular jumps of $40^{\circ}$ and $85^{\circ}$ presented as a function of number of steps.

and $85^{\circ}$, which correspond to the respective reorientations of methylene $\mathrm{C}-\mathrm{H}$ bonds in trans and cis monomers (see Figure 7), are shown in Figure 9 as a function of number of steps. It is interesting to observe that a total of four steps is sufficient in both cases for the complete loss of the original orientation. Similar calculations were performed, but not shown, for the other jump angles of the methylene $\mathrm{C}-\mathrm{H}$ bonds of Figure 7 . An average time $\tau_{0}$ for the $\mathrm{C}-\mathrm{H}$ bond OACF may be 


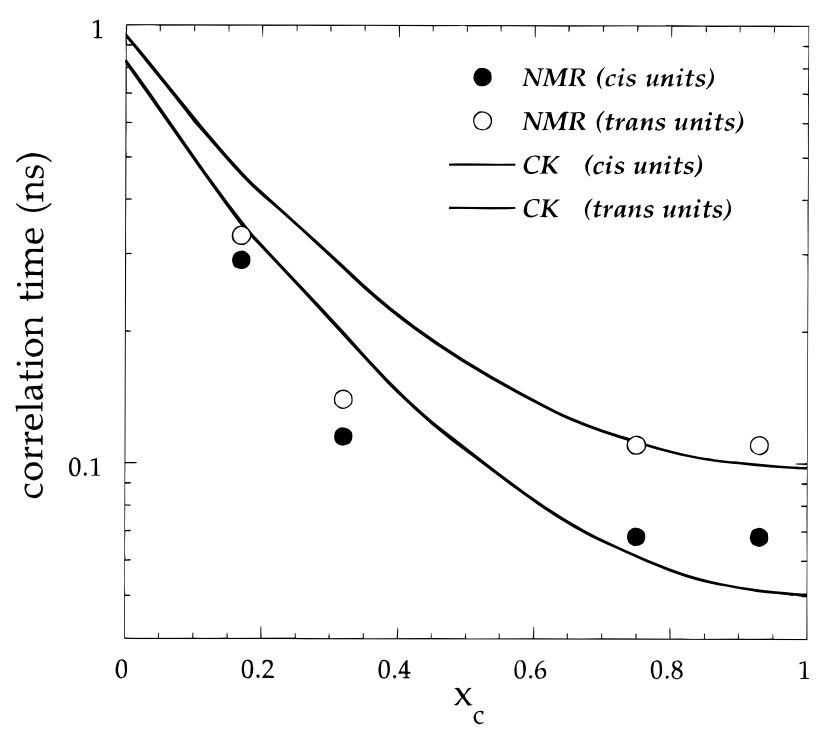

Figure 10. Comparison of correlation $\tau_{\mathrm{c}}$ obtained from the CK approach with those measured by NMR experiments. The filled and open circles represent the NMR data for the cis and trans units, respectively, in PB chains of various compositions shown along the abscissa. The curves are obtained with the CK approach.

estimated from the harmonic average $1 / \tau_{0}=\sum_{\mathrm{i}}\left(1 / \tau_{0 \mathrm{i}}\right)$, where $\tau_{0 \mathrm{i}}$ is the contribution from the ith methylene $\mathrm{C}-\mathrm{H}$ bond, assuming each $\mathrm{C}-\mathrm{H}$ bond in the close neighborhood of a rotating bond contributes by the same weight to the observed behavior. Calculations performed along these lines lead to the ratio $\tau_{0}$ (trans) $/ \tau_{0^{-}}$ (cis) $=3.4$. $\tau_{0}$ (cis) may be arbitrarily set as unity, the passage to absolute correlation times being asserted by the proportionality constant A in eq 10.

Results of calculations based on the above description are presented in Figure 10, together with the data from NMR experiments. The proportionality constant $A$ is taken as $A=0.7$, which leads to $\tau_{\mathrm{c}}$ in nanoseconds when $W_{\xi}$ is in kilocalories per mole. The lower and upper curves hold for the cis and the trans units, respectively. The filled and open circles represent the results of NMR experiments for the cis and trans units, respectively. It should be noted that only a single adjustable parameter, $A$, is used in the calculations. The composition dependence of correlation times follows that of effective friction coefficients, in general. However, in view of the dominant role of the friction coefficient and the uncertainties in the experimental data, the present comparison with experiments should be regarded as tentative. Nevertheless, the distinction between cis and trans units cannot be explained in terms of effective friction coefficients, alone. In fact, the larger amplitude orientational motions of $\mathrm{C}-\mathrm{H}$ bonds belonging to cis units, compared to those of trans units, which is revealed by the CK analysis only, explain the relative magnitudes of the correlation times of the two geometric isomers.

\section{Conclusion and Discussion}

Two major conclusions are drawn from the present CK analysis: (i) The mechanism of local motions is independent of the composition of the overall chain, but is uniquely defined by the isomeric state, cis or trans, of the repeat unit which drives a cooperative conformational motion. Accordingly, dihedral angle changes, atomic displacements, and bond reorientations in the neighborhood of a rotating bond depend uniquely on the state of the double bond of the monomeric unit compris- ing the rotating bond, irrespective of the microstructure of the chain or of the effectivefriction coefficient dictated by the microstructure. (ii) Cis units undergo larger translational and orientational displacements in response to rotameric jumps, compared to trans units. The enhanced translational and orientational degrees of freedom of cis units induce two opposing effects on the correlation times which are observed by NMR. The former is a slowing down of the relaxation process, as a consequence of the stronger frictional resistance experienced by the atoms traveling longer distances during conformational rearrangements compared to atoms belonging to trans units. The second is a faster decay of OACF S associated with the reorientation of $\mathrm{C}-\mathrm{H}$ bond vectors. The latter effect is stronger than the former, and overall the cis unit appears to have a shorter correlation time than the trans unit, which is confirmed by both NMR experiments and theory.

The CK approach permits (i) gaining an understanding of the mechanism of motion involved in local conformational transitions in polymers in the bulk state, and (ii) estimation of the relative relaxation rates of specific structural units in macromolecules. Specifically, the motions accommodating a rotational jump are found to be highly localized in PBs. These are confined to only a few bonds in the neighborhood of a rotating bond, and are consequently fully determined by the state of the double bond of the singlemonomer comprising the rotating bond, throughout a wide range of friction coefficients. It is this new concept that facilitates our interpretation of NMR data. Two factors appear to play a dominant role in determining the correlation times. First, the angular reorientation $\Delta \theta$ of the methylene $\mathrm{C}-\mathrm{H}$ bonds induced by the cooperative motions accompanying the bond isomeric jumps is an important factor explaining the faster relaxation of cis units compared to trans units in all PB chains, irrespective of the microstructure. Second, the effective friction coefficient affects the rates of relaxations of both units, its effect on cis units being slightly larger. Yet, the existing data on the monomeric friction coefficients exhibit some uncertainties, and more experimental work should be carried out for the proper determination of this parameter.

An important finding of the present study is that the cooperative segment for all compositions is basically confined to a single monomer, apart from the small rearrangements of the neighboring bonds down the chain. This is in agreement with recent molecular dynamics simulation findings of Moe and Ediger on bulk polyisoprene in which one or two monomers are stated to be involved in local conformational motions. ${ }^{25}$

A final remark is that the reorientation of a $\mathrm{C}-\mathrm{H}$ bond is not directly related to the isomerization of the neighboring $\mathrm{C}-\mathrm{C}$ bonds, again in agreement with the findings of Moe and Ediger. ${ }^{25}$ Figure 7 shows that the reorientations of the nearest methylene $\mathrm{C}-\mathrm{H}$ bonds accompanying $120^{\circ}$ torsional rotations of $\mathrm{C}-\mathrm{C}$ bonds are $75^{\circ}$ and $85^{\circ}$ for the cis unit and about $40^{\circ}$ for the trans unit. Each of the two $\mathrm{H}$ atoms tetrahedrally bonded to the rotating $\mathrm{C}-\mathrm{C}$ would exhibit a reorientation of $\pm 109.5^{\circ} / 2$ if its motion resulted solely from the $120^{\circ}$ isomerization of the $\mathrm{C}-\mathrm{C}$ bond equally distributed on both sides. The departures from the expected reorientation result from the fact that the $\mathrm{C}-\mathrm{C}$ bond undergoing the isomerization is not itself fixed in space, as demonstrated by the present CK analysis, but exhibits reorientations due to the cooperative motions accommodating the isomeric jump. 
2066 Baysal et al.

Acknowledgment. Partial support from Bogazici University Research Funds Project No. 96P003 is gratefully acknowledged. The authors thank Professor Liliane Bokobza for discussion and the communication of friction coefficient data.

\section{References and Notes}

(1) Bahar, I.; Erman, B.; Monnerie, L. Adv. Polym. Sci. 1994, $116,145$.

(2) Baysal, C.; Atilgan, A. R.; Erman, B.; Bahar, I. Macromolecules 1996, 29, 2510.

(3) Bahar, I.; Erman, B.; Monnerie, L. Macromolecules 1992, 25, 6309.

(4) Bahar, I.; Erman, B.; Monnerie, L. Macromolecules 1992, 25, 6314.

(5) Bahar, I.; Baysal, N.; Erman, B.; Monnerie, L. Macromolecules 1995, 28, 1038.

(6) Baysal, C.; Bahar, I.; Erman, B.; Monnerie, L. Macromolecules 1996, 29, 2980.

(7) Adolf, D. B.; Ediger, M. D. Macromolecules 1992, 25, 1074

(8) Ediger, M. D.; Adolf, D. B. Adv. Polym. Sci. 1994, 116, 73.

(9) Kim, E.-G.; Mattice, W. L. J . Chem. Phys. 1994, 101, 6242.

(10) Gee, R. H.; Boyd, R. H. J . Chem. Phys. 1994, 101, 8028.

(11) Moe, N. E.; Ediger, M. D. Macromolecules 1995, 28, 2329.
(12) Krajewski-Bertrand, M.-A.; Lauprêtre, F. Macromolecules 1996, 29, 7616.

(13) Chen, S. P.; Ferry, J . D. Macromol ecules 1968, 1, 270.

(14) Bokobza, L.; et al. Private communication.

(15) Abe, Y.; Flory, P. J . Macromolecules 1971, 4, 219.

(16) Van der Velden, G.; Didden, C.; Veermans, T.; Beulen, J . Macromolecules 1987, 20, 1252.

(17) Sato, H.; Takebayashi, K.; Tanaka, Y. Macromol ecules 1987, 20, 2418.

(18) Wang, H. T.; Bethea, T. W.; Harwood, H. J . Macromolecules 1993, 26, 715.

(19) Dejean de la Batie, R.; Lauprêtre, F.; Monnerie, L. Macromolecules 1988, 21, 2045.

(20) Dejean de la Batie, R.; Lauprêtre, F.; Monnerie, L. Macromolecules 1989, 22, 122.

(21) Gronski, W.; Quack, G.; Murayama, N.; Elgert, K. F. Makromol. Chem. 1975, 176, 3605.

(22) Krajewski-Bertrand, M. A.; Lauprêtre, F.; Monnerie, L. to be published.

(23) Bahar, I.; Erman, B.; Monnerie, L. Macromolecules 1990, 23, 1174.

(24) Torchia, D. A.; Szabo, A. J . Magn. Reson. 1982, 49, 107.

(25) Moe, N. E.; Ediger, M. D. Polymer 1996, 37, 1787.

MA9604707 